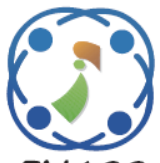

\title{
A Cloud Broker Framework for Infrastructure Service Discovery Using Semantic Network
}

\author{
Rajganesh Nagarajan $^{1 *}$ Ramkumar Thirunavukarasu $^{2} \quad$ Selvamuthukumaran Shanmugam $^{3}$ \\ ${ }^{I}$ Department of Information Technology, AVC College of Engineering, India \\ ${ }^{2}$ School of Information Technology \& Engineering, VIT University, India \\ ${ }^{3}$ Department of Computer Applications, AVC College of Engineering, India \\ * Corresponding author’s Email: anuvarajganesh@gmail.com
}

\begin{abstract}
On demand provisioning with an acceptable pricing scheme of cloud computing attracts user to enter in to the cloud arena. In this paradigm, there are many cloud service providers involved to offer different level of services and the effectiveness of the service specification, identification of services is always a challenging one. Hence, we propose a broker based cloud computing framework for enabling the cloud user to specify their service requirements in terms of numerical representation. The infrastructure type of services such as computing and storage are considered for this proposal. With respect to the user specification, the proposed broker constructs the cloud ontology to represent the available services from the service repository. With the aid of ontological representation, the proposed broker discovers the infrastructure services from the available vendor. The appropriate services are represented using semantic network which enables the user to know about the available services as per their posted requirements. Finally, the broker recommends the services with its add-on features to the cloud users. A prototype model is developed to simulate the functioning of the broker based service discovery in the cloud and the results show the performance upgradation by comparing the traditional approach.
\end{abstract}

Keywords: Cloud computing, Service discovery, Intelligent broker, Semantic network.

\section{Introduction}

Cloud computing [1] is an emerging paradigm which offers on-demand, pay-as-per usage, and utility oriented services to the user. With the advent of cloud computing, anyone can avail the required services in a rental basis. In this way, the cloud computing promotes the computing environment in three different ways such as public, private and hybrid type of cloud deployment models. In addition, this paradigm offers three major service models such as infrastructure, software and platform. The services specified in these models can be availed by the cloud user with a reasonable cost. Nowadays, there are lot of cloud service providers such as Amazon, Rackspace, Salesforce are available to provide the needed services for their customers. Due to the increasing number of cloud service providers, it is essential to ensure the services and its effectiveness for the benefit of cloud users. Likewise, a major problem faced by the cloud user is the selection of right services from the providers. Most of the time, the cloud users are failed to pick their services because of their inexperience in the service discovery process.

In cloud computing, service discovery [2] is to find a suitable cloud service that essentially meet out the cloud user requirement in the aspect of functional, non-functional and the budgetary constraints. Without considering these factors, it is not possible to discover the right services from the multiple cloud providers. As a solution, a cloud broker is introduced to act as a mediator between the provider and user for smoothing the process of service discovery. Unfortunately, many of the service discovery schemes are unable to perform the right discovery of services due to the issues such as, (i) incorrect / 
invalid service details, (ii) handling the uncertainties in service specification, (iii) failed to make interaction between the multiple cloud providers, (iv) handling of the trusted third parties feedbacks, (v) user / service based discovery, (vi) dynamic adaptation of cloud user's feedback, and (vii) issues with respect to the service upgradation for future use.

Though many of the works [3-9] with respect to the cloud service discovery are expressed the capabilities and action list of the broker in service discovery, certain challenges for the broker based cloud are still exist. To cater this, an intelligent broker must be constructed for pre-processing, ranking, and constructing requirement ontology for the automated service discovery process. Whenever a new service is hosted, the broker must evaluate them against the user's need based on well-known soft computing benchmark such as Artificial intelligence, Neural networks, Fuzzy model, and Supervised learning techniques. This circumstance prompts the need and inclusion of a new broker for the cloud environment. We have designated such a broker as an 'intelligent broker' to perform various roles more than the existing one.

Our contribution towards to the broker based cloud service discovery are summarized as follows:

Proposing an Intelligent Broker (IB) with a constructive end-user portal to define the service requirements very precisely in terms of numerical values.

(ii) Representing the knowledge in the form of ontology to perform the reasoning process.

(iii) Discovering the appropriate IaaS type cloud services with the aid of constructed cloud ontology and represent them in the form of semantic networks. By performing the intersection search, the reasoning process is effectively performed.

(iv) A simulation platform is designed and developed to analyse the broker performance with the existing approaches.

The rest of this paper is structured as follows. Section 2 presents the related works with respect to the service discovery in cloud computing. Section 3 express our proposal with the semantic network representation. A newly developed algorithm for the service discovery and recommendation is also presented. In section 4, we have simulated our proposal with its performance. Finally, section 5 concludes our work with future works.

\section{Related work}

Since cloud computing is an emerging field, there are many cloud services are published by the cloud service providers. Similarly, the need for the cloud brokers with the intelligence feature is demanded from the cloud user side for the effective discovery of cloud services. To make a brief note on this, this section highlights the previous work of various researchers. Generally, state-of-art cloud service selection approaches can be classified into two major categories namely, ontology based ranking and multi criteria based service selection.

Han \& Sim [10] developed an agent to discover the services and reduced the unwanted surfing process. Here, the authors used the ontology for representing the services. But, the proposed mechanism failed to compare the nature of services with respect to the service utility and functional requirements must be considered for the improved service rating. Martens \& Teuteberg [11] insisted the need and necessity of a formal decision-making tool which accounts both cost and risk. Liew et al. [12] formed a P2P network with a set of service providers and allowed them to advertise and share their services among the grouped users. Hence, the providers are responsible for their service advertisement and maintain the directory node for the service details.

Zhang et al. [13] proposed an ontology-based approach which tackled the complexities of the dynamic nature of cloud computing. Also, the proposed ontology has been pre-meditated to confine static and dynamic QoS arrangement on the infrastructure layer. Unfortunately, the approach did not employ any suitable mechanism to face the multiple conflicting objectives of the cloud service user. Noor et al. [5] introduced a Cloud Service Crawler Engine (CSCE) to perform the service selection. Their work constructed the ontology, then performed the discovery, validation, and categorization of cloud services. With the introduction of ontology, service discovery and the filtering process have been simplified.

Nagireddi \& Mishra [14] discussed about the importance of ontology in cloud service selection. Also, the authors have constructed the cloud ontology as the database, which serves as a repository for various service offered by cloud service providers. The problem with this approach is that the individual provider is responsible for registering with the 'intercloud registry'. Their proposal lacks in dealing the issue of automatic service findings. Qian et al. [15] developed a model for discovering the IaaS type of services from the cloud service providers. Apart from the parameters like cost and performance, they have incorporated geographical location of the service for the proper interaction between the provider and user. But, this paper fails to ensure the reliability of location based service discovery with respect to the 
cloud consumer. Rodriguez-Garcia et al. [16] insisted the use of semantics for optimizing the search process. The authors introduced the automation method for assisting the user in the process of adding of metadata to empower the cloud services descriptions. In addition, they build a semantic search engine to improve the precision and recall measures of the search results. The proposed work supports the query based requirements in the service selection. Joshi et al. [17] proposed their approach for service discovery and provisioning of public type cloud services. The service selection is limited to the public type of cloud only. So, it addresses the problem for selecting the private type of cloud services.

Mastroianni \& Papuzzo [18] focused their approach in improving the effectiveness of service discovery. Their proposal clustered the service descriptors based on their frequency value. By the way of clustering, the co-occurrences of services, response time, bandwidth, and processing load are significantly reduced. This paper broadly discussed the mobile agents and its functioning in service discovery. Zhygmanovskyi \& Yoshida [19] presented a detailed study in service provisioning. They proposed a P2P technology to perform discovery and sharing of services with the Distributed Hash Table (DHT) approach, which guaranteed the accuracy and reliability of the underlying P2P model. Also, it allows sliding doors between the service description areas to execute an ample range of queries. A proper enhancement has been needed to initiate the fully automated service discovery process in the cloud. Wang et al. [6] proposed an adaptive learning based cloud broker for the dynamic cloud service selection. In their work, they have proposed several algorithms to depict the process of their system. Also, they have reviewed the previous work and concluded that their work concentrates on the dynamic selection rather than the static. From this paper, the role of Artificial Intelligence has been highlighted in the construction of the cloud broker.

Lin et al. [20] proposed a broker based on indexing tree (B-Tree) structure for the selection of cloud services. The authors have concentrated more on the handling of service information rather than identifying the right services. The tree had expanded whenever the providers were increased. Hence, the representation of the service information was very complex. Sim [21] introduced the agent paradigm to build the software tools and testbeds for managing the cloud resources. With this, the author has developed 'Cloudle' - an agent based cloud service search engine with reasoning capabilities to select the cloud services. Accordingly, the challenges such as handling of dynamic services, customers preferences with respect to their mindset are not addressed.

Li et al. [22] presented a broker based cloud service selection verification system to ensure the right cloud service selection. The authors have developed an index structure using Merkle hash tree to ensure the authenticity, satisfiability and the completeness of the service selection results. The construction of hash tree has become a difficult task when the number of request as well as results are increased. Kang and Sim [23] developed an agent based cloud service discovery protocol based on cloud ontology. They have improved their old work $[8-9,24]$ with respect to the agent's part and their moto was based on various kinds of reasoning process. In addition to that, the authors have used multiple broker agents to deal the problem of scalability. Alfazi et al. [25] proposed a cloud service search engine that has the capability to automatically identify cloud services in real environments. The developed search engine can detect cloud services effectively from the Web sources. Furthermore, the authors have focused on learning the cloud service features, such as similarity function, semantic ontology and cloud service components to identify the cloud services. Shetty and D'Mello [26] proposed an approach of hybrid discovery using the wellstructured repository. In the data representation model, the authors represented the semantic information, which is in the form of xml. Instead of user-centric repository, the system might have to be improved to cover the providers and the broker also.

Chainbi [27] attempted the cloud service discovery using multi-criteria approach. The cloud users can select their services from the multiple cloud providers. The sorted lists of preferred services are displayed with the calculated scores for their recommendations. With this proposal, the authors enriched the consumer's capability for discovering the services based on weighted scores. Ramacher and Monch [28] proposed a hierarchical approach for the service selection and minimized the cost and maximized the quality of services. But, a huge computational problem was existing with this proposed model while considering the uncertain requirements. Bosc et al [4] proposed a fuzzy logic based model to perform the service selection with the functionalities such as query transparency, parametric semantics, and ranked service with synergy management. Hence, the problem stated in [3] about the ranking of services has been rectified in this approach. Qu et al. [29] proposed a fuzzy based service selection model by considering the thirdparty assessment against the customer requirement. There was no such common mechanism found for the 
trustworthiness of the testing party's result. Nie et al. [30] used the Analytic Hierarchy Process (AHP) to calculate the weights for security, cost and reputation factors to perform the service discovery process. However, the approach was unable to handle the QoS related criteria in its hierarchy, so it increased the complexity of decision-making process. Also, the authors have not focused the monitoring process in the effective discovery of cloud service. Karim et al. [31] proposed a technique with the aid of AHP in the service discovery arena and provide the guaranteed services for the cloud consumer. Here, the services are ranked as per their QoS weight. The need of cloud service broker accounted here is open for the future work.

\section{Proposed model with an intelligent broker framework}

A cloud broker [32] is a software application which is designed to serve as an umpire between the consumer and provider. Although the cloud broker offers various value-added services on behalf of the parties, it is more appropriate to build the broker with the inclusion of intelligent computational techniques. Such kinds of techniques [33] facilitate intelligent reasoning and decision-making capability to the broker $[34,35]$. From our perspective, the intelligent broker is defined as, "a software entity based on the cloud brokerage model that offer cloud services with the aid of computational intelligence techniques such as artificial intelligence, soft computing, machine learning and data mining towards providing a platform for both cloud users and providers". The proposed broker architecture contains (i) user interface, (ii) cloud ontology, (iii) service discovery and (iv) IaaS service repository is shown in Fig. 1.

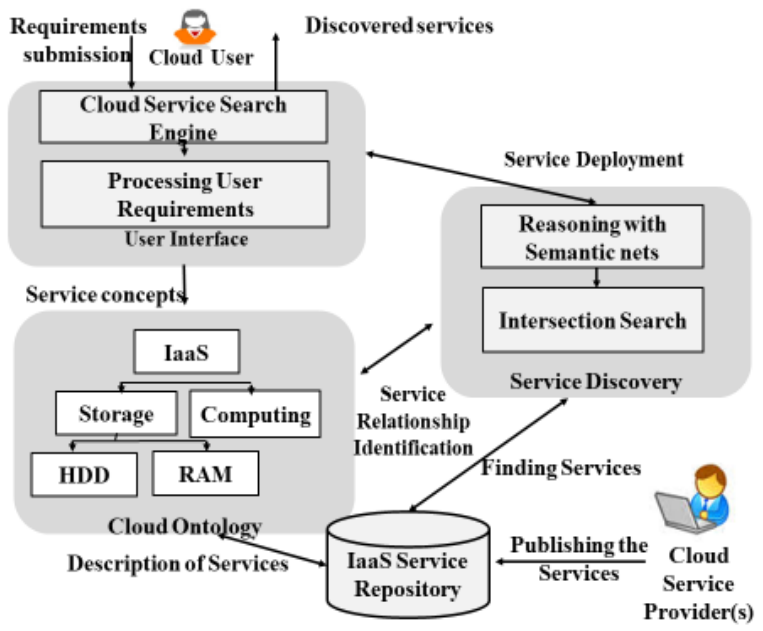

Figure. 1 Proposed system with intelligent broker framework

\subsection{User interface}

The proposed broker architecture enables the cloud user to interact via this portal. It is planned to obtain the service requirements in terms of numerical value with the aid of 'cloud service search engine' and then pre-process the input values with respect to the providers service specification details. The processed information is termed as 'service concepts' and given as the input for the construction of cloud ontology. The posted input requirements of three cloud users are shown in Table 1.

\subsection{Cloud ontology}

An ontology [36] is a description of different cloud concepts with its relationships to facilitate the reasoning among the all types of cloud services. It enables the users/machines to process the information more precisely and conveniently. With respect to our scenario, the cloud broker constructs a cloud ontology for the effective representation of IaaS types of cloud services with reasonable budget. With respect to the posted requirements (Table 1), the broker constructs the ontologies (Fig. 2) by referring the service descriptions of various cloud service providers.

Table 1. Requirements specification by the cloud users

\begin{tabular}{|c|c|c|c|c|}
\hline $\begin{array}{c}\text { Cloud } \\
\text { user } \\
\text { (CU) }\end{array}$ & $\begin{array}{c}\text { Computing } \\
\text { services } \\
(\mathrm{GHz})\end{array}$ & $\begin{array}{c}\text { RAM } \\
\text { (GB) }\end{array}$ & $\begin{array}{c}\text { HDD } \\
\text { (TB) }\end{array}$ & $\begin{array}{c}\text { Expected } \\
\text { budget }\end{array}$ \\
\hline CU1 & 3.2 & 4 & 1 & $\$ 0.25$ \\
\hline CU2 & 3.0 & 2 & 1 & $\$ 0.15$ \\
\hline CU3 & 3.4 & 8 & 2 & $\$ 0.30$ \\
\hline
\end{tabular}

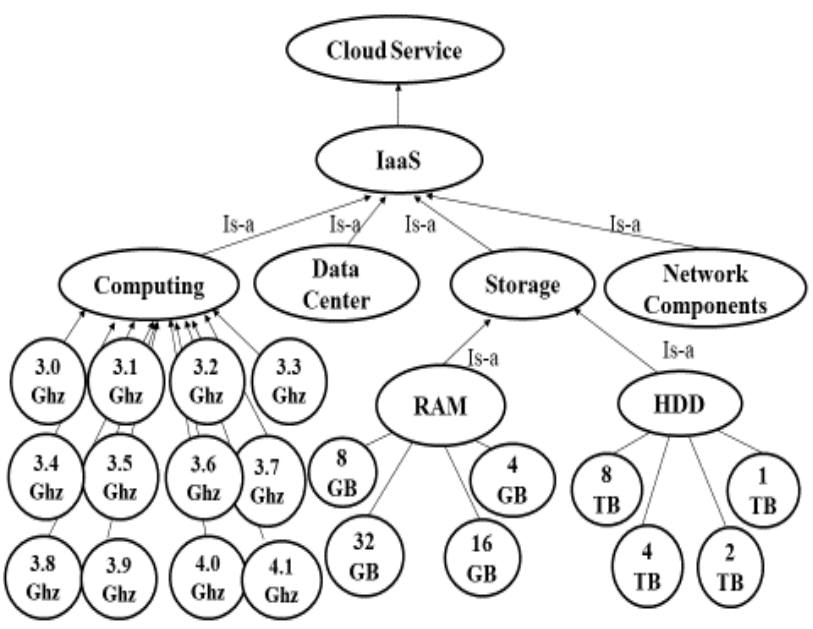

Figure. 2 Ontological representation of IaaS cloud services 


\subsection{Service discovery}

Service discovery in cloud computing leads to find a suitable cloud service that essentially refers to the discovery of service description. A service description contains the functional, non-functional capabilities along with the characteristic of a service. The discovery is constrained by functional, technical specifications of the services, and the budgetary constraints with appropriate security policies of the services are called as 'service concepts'. The meaning of service concepts such as its connectivity among the available service instances are represented in the form of semantic nets. Here, the service entities are represented as a set of nodes connected to each other by a set of labelled arcs for representing the relationship among the discovered services. The Algorithm 1 illustrates the general view of service discovery process in clouds.

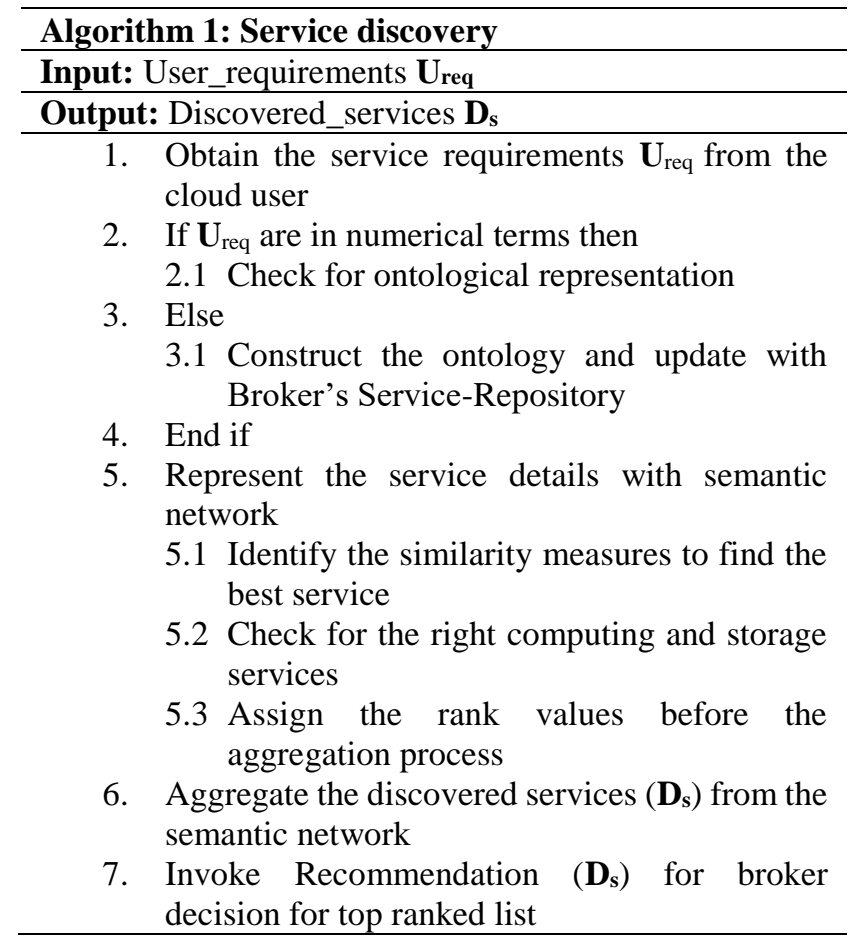

A typical semantic network with the discovered IaaS service instances is shown in Fig.3. The broker applies intersection search to discover the similarities between the discovered services. The similarities between the posted requirements with the provider's service description is determined in a way such that, for all the posted requirements of cloud user CU1 are checked against with the service collection of cloud provider CPs. Let $\mathrm{A}(\mathrm{x})$ is the service requirements of a cloud user and $\mathrm{B}(\mathrm{x})$ is the available services of some cloud providers, then the similarities are determined by,
$S_{A, B}=|A(x) \cap B(x)| / A(x)$

In another way, the service similarities can also be determined by,

$S_{B, A}=|B(x) \cap A(x)| / B(x)$

For example, the Figure.2 shows the ontological representation for the IaaS type of services in a different level of hierarchy. By considering the service requirements for the computing services of cloud user 'CU1', the similarities between the services are represented as;

$S($ Computing $) \rightarrow S(3.0 \mathrm{Ghz})=2, S(3.1 \mathrm{Ghz})=2, S(3.2$ $G h z)=2, S(3.3 G h z)=2, S(3.4 G h z)=3, S(3.5 G h z)=3$

$$
\rightarrow S(3.2 \mathrm{Ghz}, 3.3 \mathrm{Ghz})=2 / 3
$$

Likewise, for the RAM service, the similarities are represented from the Fig. 2 is,

$S(R A M) \rightarrow S(4 G B)=4, S(8 G B)=4, S(16 G B)=4$, $S(32 G B)=4$

$\rightarrow S(4 G B, 8 G B, 16 G B, 32 G B)=4 / 4$

Consider the above-mentioned example, the broker lists out the possible cloud services along with the providers which are very closer to the posted requirements of user U1 (Table 2).

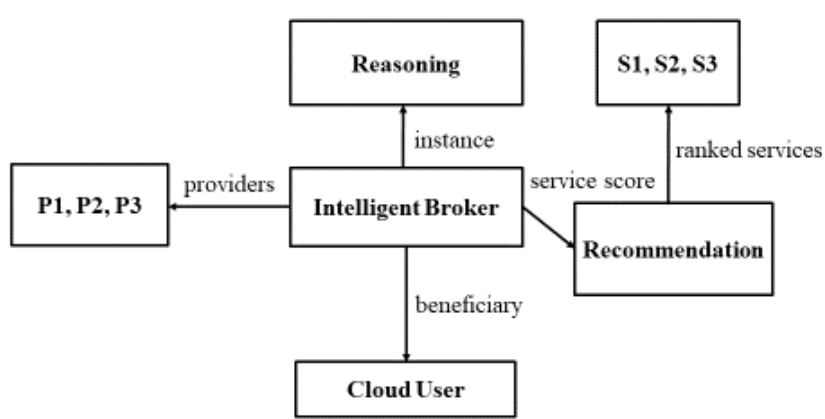

Figure. 3 Semantic representation of discovered services

Table 2. Discovered services for the user U1

\begin{tabular}{|c|c|c|c|c|}
\hline $\begin{array}{c}\text { Cloud } \\
\text { service } \\
\text { provider } \\
\text { (P) }\end{array}$ & $\begin{array}{c}\text { Computing } \\
\text { services } \\
\text { (GHz) }\end{array}$ & $\begin{array}{c}\text { RAM } \\
(\mathrm{GB})\end{array}$ & $\begin{array}{c}\text { HDD } \\
\text { (TB) }\end{array}$ & $\begin{array}{c}\text { Exact } \\
\text { budget }\end{array}$ \\
\hline P1 & 3.2 & 4 & 2 & $\$ 0.25$ \\
\hline P2 & 3.2 & 2 & 1 & $\$ 0.20$ \\
\hline P3 & 3.3 & 4 & 1 & $\$ 0.20$ \\
\hline
\end{tabular}


The best selection is up to the decision of intelligent broker by considering the general merits and cost factors of the service provider. Accordingly, 'P1' and 'P3' have been identified with the aid of constructed ontology, the 'P1' has been recommended to the user as a first choice by considering the budgetary requirements of the user. Though 'P1' service cost stands above the stated budgetary requirements of the user, their desirable service feature ( 2 TB of HDD) has been weighted and recommended to the user (Table 3). The Algorithm 2 shows the broker decision about the top most service selection from the discovered services.

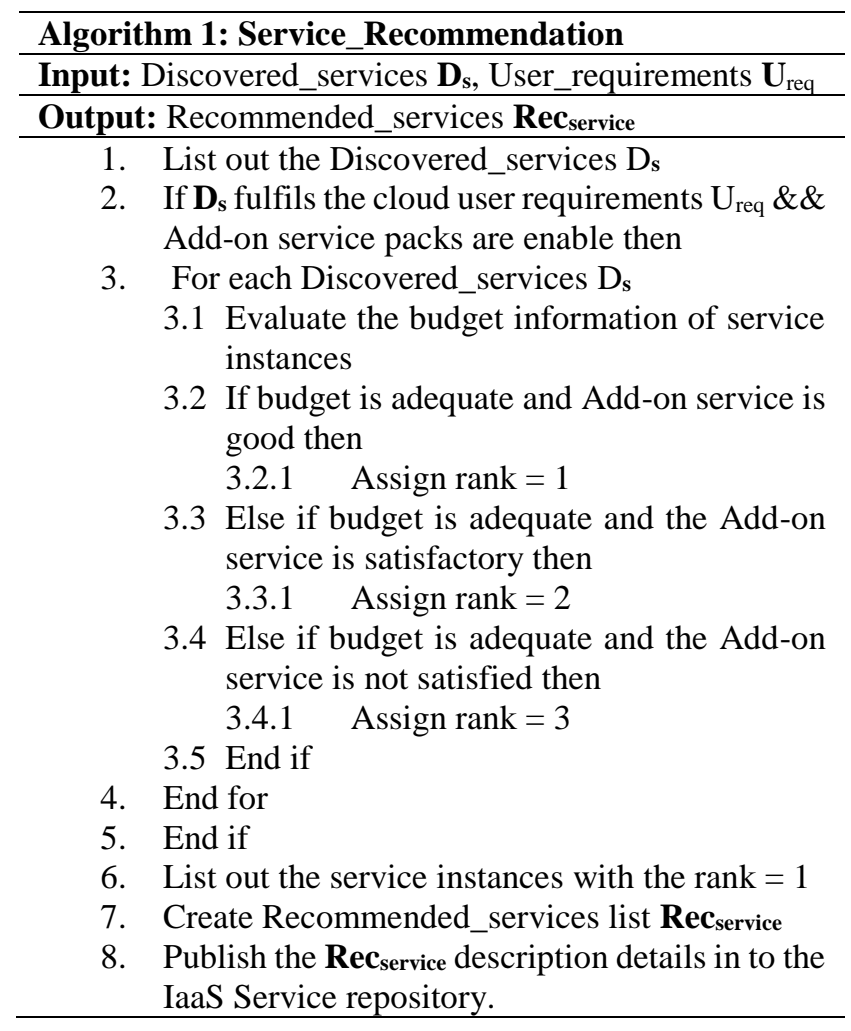

Table 3. Recommended IaaS services with features

\begin{tabular}{|l|c|c|}
\hline \multicolumn{1}{|c|}{ Instance Details } & \multicolumn{2}{|c|}{$\begin{array}{c}\text { Instance features with add-on } \\
\text { services }\end{array}$} \\
\hline $\begin{array}{l}\text { Cloud service } \\
\text { provider }\end{array}$ & P3 & P1 \\
\hline CPU & 3.2 & 3.3 \\
\hline RAM & 4 & 4 \\
\hline HDD & 1 & 2 \\
\hline Customer support & $\sqrt{ }$ & $\sqrt{ }$ \\
\hline Trustworthiness & $\sqrt{ }$ & X \\
\hline Availability & $\sqrt{ }$ & $\sqrt{ }$ \\
\hline Service updates & $\sqrt{ }$ & 0.25 \\
\hline $\begin{array}{l}\text { Feedback } \\
\text { responses }\end{array}$ & $\sqrt{ }$ \\
\hline Budget & 0.20 & \\
\hline
\end{tabular}

In this perspective, our proposed intelligent broker performs service recommendations besides service matching. In the given example, we have depicted how intelligent broker performs and recommends services from a single cloud provider. Also, the proposed broker periodically investigates the services and creates a service log to ensure the service effectiveness and their business levels. Such a self-healing attitude plays a vital role in performing effective customer relationship management.

\subsection{IaaS service repository}

All kinds of IaaS type cloud services with its service descriptions are published with the service repository. Whenever a service request comes from the cloud user, the broker checks for the repository after constructing the cloud ontology. From the cloud service provider's perspective, the repository plays a vital role by incorporating the different type of services. In another way, our broker is responsible to identify the appropriate services on behalf of the cloud user.

\section{Simulation and results}

To begin with, we have developed a .NET based prototype to demonstrate the cloud broker functionality with respect to the discovery of cloud infrastructure services. The developed model simulates the functioning of our idea by taking the sample data set. We have considered 100 service instances from 10 cloud service providers. Each service instances are in turn represented as a set of facilities such as computing, RAM and Hard Disk Drive (HDD). The tests were conducted using a HP Pavilion Laptop, with 8GB of RAM, 1 TB of Hard drive and a Intel (R) Core (TM) i7-6500 CPU (2.59 $\mathrm{GHz}$ ) processor. Fig. 4 shows the broker interface for supporting the cloud provider and user for service publishing and discovery process. Whenever the new services are emerged, it can be updated with the 'Service Repository' of the broker. Fig. 5 shows the new/updated service publishing with the broker. So, the cloud user can get the updated service instances with respect to their timely need. Similarly, as per the posted service requirements of cloud user ' $\mathrm{CU} 1$ ', the discovered services with their ranking is presented in Fig. 6. Also, the broker recommends the top ranked services with respect to its budgetary details. Instead of just recommending the services to the user, the proposed broker continuously assists them in all aspects with respect to the mutual binding of cloud service provider. 


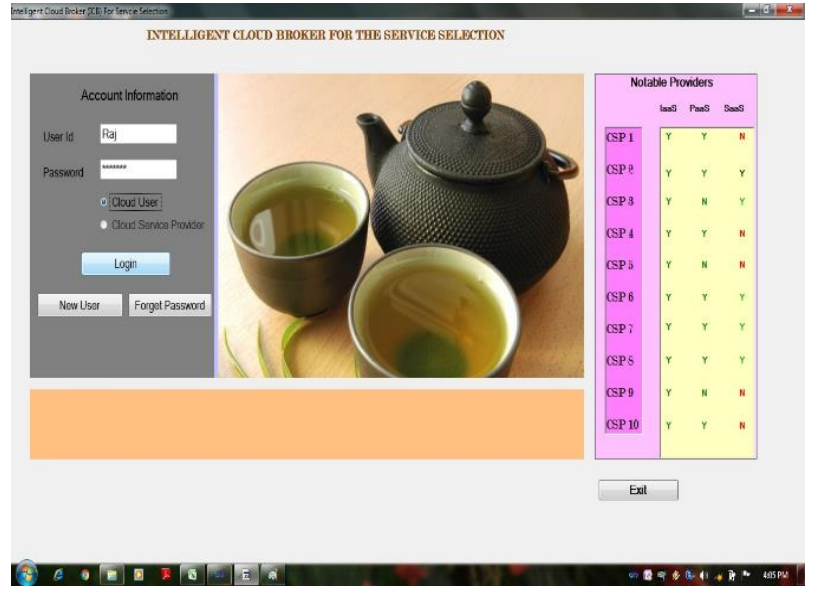

Figure. 4 Proposed cloud broker for cloud provider and user

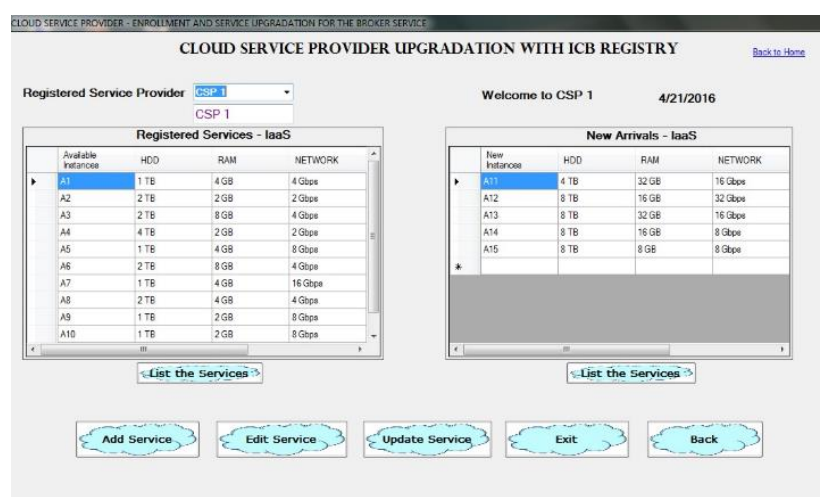

Figure. 5 Service publication by the cloud service provider

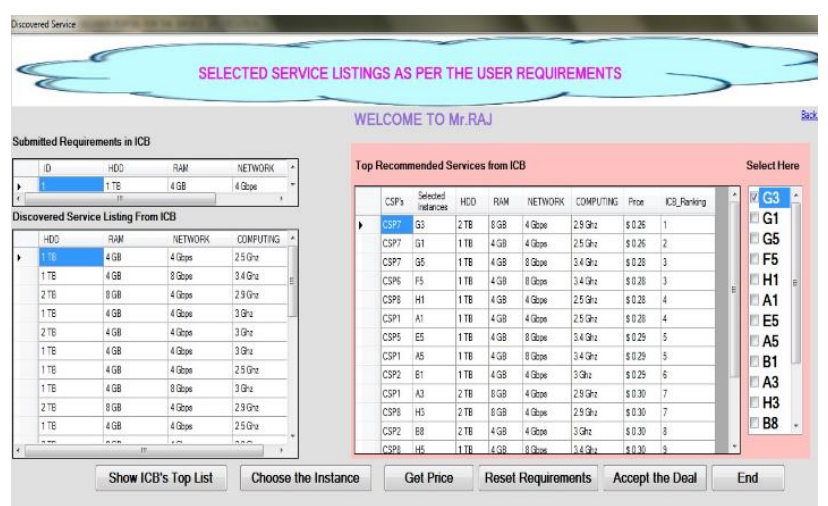

Figure. 6 Services discovered by the cloud broker

Apart from the budgetary constraints, how the broker is ready to give its uninterrupted support in terms of trustworthiness of service, availability, service updates, responses to the query are also considered during the provisioning of services. Simply these are termed as value-added services with a minimal charge. As a result, we have compared our proposal with an existing clustering based service discovery system to show the performance.

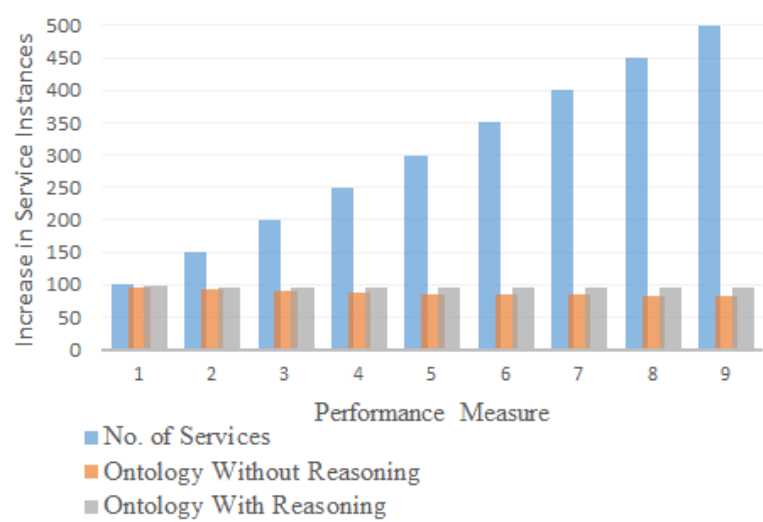

Figure. 7 Service discovery with and without reasoning process

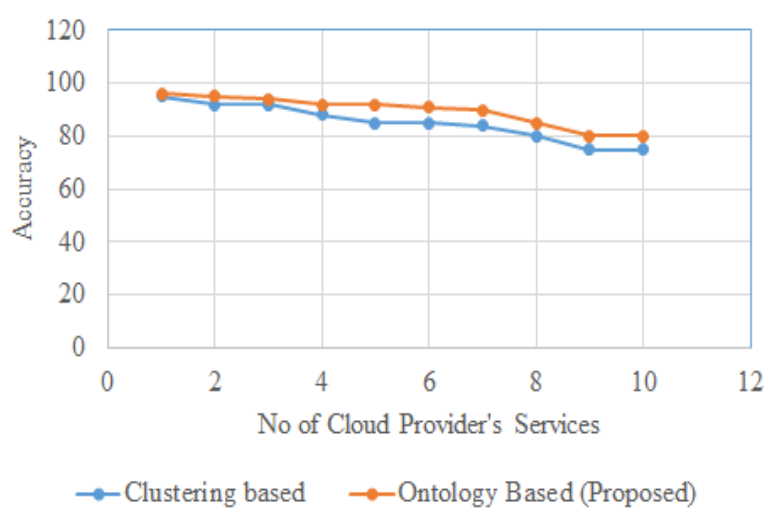

Figure.8 Accuracy in terms of service representation and selection

In [37], the functionally equivalent services are resulted as dissimilar because of proposed matching process. Also, the oppositeness relationship among the service concepts are calculated based on subjective assessment instead of reasoning process, which may be inaccurate. Similarly, the construction of abstract network in [38] increases the complexity of similarity identification and resulted to delaying the process of service selection and composition. In our proposal, the semantic network is used to represent the similar services with the aid of constructed ontology. Hence, the proposed system improves the overall performance by reducing the time needed to construct the ontology and semantic network based service representation. From the experimental work, it is clearly identified that the increased service instances with multiple copies without reasoning process resulted the performance degradation in the existing methods (Fig. 7). Also, the construction of ontology with the service concepts and the discovery of services through the developed broker certainly improves the system performance. 
Because of the semantic network representation of recommended service instances in the final stage, our proposal certainly reduces the memory requirements with respect to the service details management. The usage of intersection search in the process of service representation with the semantic network increased the overall performance of the system is shown in Fig. 8.

\section{Conclusion}

In this paper, we present an intelligent cloud broker for the service discovery from the multiple cloud service providers. The proposed system obtains the numerical service requirements for the infrastructure type of cloud services and constructs the ontology for reasoning purpose. In this paper, two algorithms are proposed to support the service discovery and recommendation process. First, the broker discovers the appropriate services as per the posted service requirements and represent them using semantic network. Second, the intersection search is carried out to recommend the ranked services for the cloud user. We have developed a .NET based prototype model to demonstrate our proposal with the predefined set of data. At first, the service discovery process is carried out without the reasoning process and the improvement with the inclusion of reasoning is recorded. Likewise, the other experiment resulted the improved accuracy in terms of service representation / selection by comparing the existing methods. Finally, the cloud broker recommends a set of infrastructure services with its add-on features as per the expected budgetary constraints. In future, we would like to strengthen our broker for receiving the user's requirements without ambiguity and simplifies the service specification through the linguistic representation.

\section{References}

[1] R. Buyya, J. Broberg, and A.M. Goscinski, Cloud computing: Principles and paradigms, John Wiley \& Sons, Vol.87, 2010.

[2] F. Zeshan, R. Mohamad, M.N. Ahmad, S.A. Hussain, A. Ahmad, I. Raza, A. Mehmood, I. Ulhaq, A. Abdulgader, and I. Babar, "Ontologybased service discovery framework for dynamic environments", IET Software, Vol.11, No. 2, pp.64-74, 2017.

[3] E. Damiani and M.G. Fugini, "Dynamic service identification in a distributed environment", JACIII, Vol.3, No.5, pp. 401-408, 1999.

[4] P. Bosc, E. Damiani, and M. Fugini, "Fuzzy service selection in a distributed object-oriented environment", IEEE Transactions on Fuzzy Systems, Vol.9, No.5, pp.682-698, 2001.

[5] T.H. Noor, Q.Z. Sheng, A. Alfazi, A.H. Ngu, and J. Law, "CSCE: a crawler engine for cloud services discovery on the world-wide web", In: Proc. of International Conf. on Web Services (ICWS), pp.443-450, 2013.

[6] X. Wang, J. Cao, and Y. Xiang, "Dynamic cloud service selection using an adaptive learning mechanism in multi-cloud computing", Journal of Systems and Software, Vol.100, pp.195-210, 2015.

[7] S. Wang, W. Shen, and Q. Hao, "An agent-based web service workflow model for inter-enterprise collaboration", Expert Systems with Applications, Vol.31, No.4, pp.787-799, 2006.

[8] J. Kang and K.M. Sim, "Cloudle: An agent-based cloud search engine that consults a cloud ontology", In: Proc. of International Conf. on Cloud Computing and Virtualization, 2010.

[9] J. Kang and K.M. Sim, "Towards agents and ontology for cloud service discovery", In: Proc. of International Conf. on Cyber-Enabled Distributed Computing and Knowledge Discovery (CyberC), pp.483-490, 2011.

[10] T. Han and K.M. Sim, "An ontology-enhanced cloud service discovery system", In: Proc. of International Multi Conf. on Engineers and Computer Scientists, Vol.1, No.2010, pp.17-19, 2010.

[11] B. Martens and F. Teuteberg, "Decision-making in cloud computing environments: A cost and risk based approach", Information Systems Frontiers, Vol.14, No.4, pp.871-893, 2012.

[12] S.Y. Liew, E.N. Teoh, P.Y. Lau, P.K. Chong, A. Nazir, E.K. Karuppiah, and Y.M. Yassin, "An overlay approach for service discovery in a largescale decentralized cloud", In: Proc. of Asia Pacific Cloud Computing Congress (APCloudCC), pp.68-71, 2012.

[13] J. Zhang, J. Wang, P.C. Hung, Z. Li, J. Liu, and K. He, "Leveraging Fragmental Semantic Data to Enhance Services Discovery", Information System Frontiers, 2012.

[14] V.S.K. Nagireddi and S. Mishra, "A naive approach for cloud service discovery mechanism using ontology", In: Proc. of National Conf. on Parallel Computing Technologies, pp.1-7, 2013.

[15] H. Qian, H. Zu, C. Cao, and Q. Wang, "CSS: Facilitate the cloud service selection in IaaS platforms", In: Proc. of International Conf. on Collaboration Technologies and Systems (CTS), pp.347-354, 2013.

[16] M.A. Rodriguez-Garcia, R. Valencia-Garcia, F. Garcia-Sanchez, and J.J. Samper-Zapater, 
"Ontology-based annotation and retrieval of services in the cloud", Knowledge-Based Systems, Vol.56, pp.15-25, 2014.

[17] K.P. Joshi, Y. Yesha, and T. Finin, "Automating cloud services life cycle through semantic technologies", IEEE Transactions on Services Computing, Vol.7, No.1, pp.109-122, 2014.

[18] C. Mastroianni and G. Papuzzo, "A selforganizing P2P framework for collective service discovery", Journal of Network and Computer Applications, Vol.39, pp.214-222, 2014.

[19] A. Zhygmanovskyi and N. Yoshida, "Cloud service provisioning based on peer-to-peer network for flexible service sharing and discovery", Journal of Computer and Communications, Vol.2, No.10, pp.17, 2014.

[20] D. Lin, A.C. Squicciarini, V. N. Dondapati, and S. Sundareswaran, "A cloud brokerage architecture for efficient cloud service selection", IEEE Trans. on Services Computing, No.99, pp.1, 2016.

[21] K.M. Sim, "Agent-based cloud computing", IEEE Transactions on Services Computing, Vol.5, No.4, pp.564-577, 2012.

[22] J. Li, A. Squicciarini, D. Lin, S. Sundareswaran, and C. Jia, "MMBcloud-tree: Authenticated index for verifiable cloud service selection", IEEE Transactions on Dependable and Secure Computing, 2015.

[23] J. Kang and K.M. Sim, "Ontology-enhanced agent-based cloud service discovery", International Journal of Cloud Computing, Vol.5, No.1-2, pp.144-171, 2016.

[24] J. Kang and K.M. Sim, "A cloud portal with a cloud service search engine", In: Proc. of International Conf. on Information and Intelligent Computing IPCSIT, Vol.18, 2011.

[25] A. Alfazi, Q.Z. Sheng, W.E. Zhang, L. Yao, and T.H. Noor, "Identification as a Service: LargeScale Cloud Service Discovery over the World Wide Web", In: Proc. of International Congress on Big Data (BigData Congress), pp.485-492, 2016.

[26] J. Shetty and D.A. D'Mello, "An XML based data representation model to discover infrastructure services", In: Proc of International Conf. on Smart Technologies and Management for Computing, Communication, Controls, Energy and Materials (ICSTM), pp.119-125, 2015.

[27] W. Chainbi, "A multi-criteria approach for web service discovery", Procedia Computer Science, Vol.10, pp.609-616, 2012.

[28] R. Ramacher and L. Monch, "Service selection with runtime aspects: A hierarchical approach",
IEEE Transactions on Services Computing, Vol.8, No.3, pp.481-493, 2015.

[29] L. Qu, Y. Wang, M.A. Orgun, L. Liu, H. Liu, and A. Bouguettaya, "CCCloud: Context-aware and credible cloud service selection based on subjective assessment and objective assessment", IEEE Transactions on Services Computing, Vol.8, No.3, pp.369-383, 2015.

[30] G. Nie, Q. She, and D. Chen, "Evaluation index system of cloud service and the purchase decision-making process based on AHP”, In: Proc. of International Conf. on Informatics, Cybernetics, and Computer Engineering (ICCE2011), pp.345-352, 2011.

[31] R. Karim, C. Ding, and A. Miri, "An end-to-end QoS mapping approach for cloud service selection", In: Proc. of Ninth World Congress on Services, pp.341-348, 2013.

[32] N. Rajganesh and T. Ramkumar, "A review on broker based cloud service model", CIT. Journal of Computing and Information Technology, Vol.24, No.3, pp.283-292, 2016.

[33] S.G. Grivas, T.U. Kumar, and H. Wache, "Cloud broker: Bringing intelligence into the cloud", In: Proc. of International Conf. on Cloud Computing, pp.544-545, 2010.

[34] N. Rajganesh, T. Ramkumar, and S. Selvamuthukumaran, "A fuzzy logic based trust evaluation model for the selection of cloud services", In: Proc. of International Conf. on Computer Communication and Informatics, 2017.

[35] R. Nagarajan, R. Thirunavukarasu, and S. Shanmugam, "A fuzzy-based intelligent cloud broker with MapReduce framework to evaluate the trust level of cloud services using customer feedback", International Journal of Fuzzy Systems, Vol. 20, No. 1, pp. 339-347, 2018.

[36] B. Karim, Q. Tan, J.R. Villar, and E. De la Cal, "Resource brokerage ontology for vendorindependent Cloud Service management", In: Proc. of International Conf. on Cloud Computing and Big Data Analysis (ICCCBDA), pp.466-472, 2017.

[37] F. Chen, M. Li, H. Wu, and L. Xie, "Web service discovery among large service pools utilising semantic similarity and clustering", Enterprise Information Systems, Vol.11, No.2, pp.452-469, 2017.

[38] F. Li, L. Zhang, Y. Liu, Y. Laili, and F. Tao, "A clustering network-based approach to service composition in cloud manufacturing", International Journal of Computer Integrated Manufacturing, pp.1-12, 2017. 